\title{
Second-order spectrum extraction based on ionospheric clutter
}

\author{
Anyun Yin ${ }^{\text {a) }}$ and Zili $\mathbf{L i}^{\text {b) }}$ \\ School of Electronic Engineering, GuangXi Normal University, \\ Guilin City, the Guangxi Zhuang Autonomous Region, 541004, China \\ a)foodieanan@163.com \\ b) zlienishi@mailbox.gxnu.edu.cn
}

\begin{abstract}
The reflection and scattering effects of ionosphere can cause clutter interference to the receiving signal of high frequency surface wave radar (HFSWR), which makes it difficult to analyze the spectrum of signal echo. In order to separate the second-order spectrum with wave information component from spectrum containing ionospheric clutter interference, we propose a hybrid echo signal processing algorithm. That is, analyzing the ionospheric signal characteristics for a specific water's latitude and on the basis of the existing elimination algorithm, according to the characteristics of large scale steady state of the ocean, we introduce parameters such as coherence time and distance value, making an intensive study of the diving method of second-order spectrum area, and obtaining a new extraction algorithm with regional characteristics and strong pertinence. Experimental results show that this method is effective.
\end{abstract}

Keywords: ionospheric clutter, mixed echo, signal processing, specific sea area, second-order spectrum extraction

Classification: Fundamental Theories for Communications

\section{References}

[1] S. T. Gille, "An introduction to ocean remote sensing," Eos Trans. Am. Geophys. Union, vol. 86, no. 12, p. 125, 2005. DOI:10.1029/2005EO120009

[2] R. Shah and J. L. Garrison, "Application of the ICF coherence time method for ocean remote sensing using digital communication satellite signals," IEEE J. Sel. Topics Appl. Earth Observ. in Remote Sens., vol. 7, no. 5, pp. 1584-1591, 2014. DOI:10.1109/JSTARS.2014.2314531

[3] B. Zhang and Y. J. He, "Research progress of the ocean remote sensing information extraction technology under high sea states," J. Ocean Technol., 2015.

[4] W. Huang and E. W. Gill, "An alternative algorithm for wave information extraction from X-band nautical radar images," IET International Radar Conference 2013, pp. 1-5, 2013. DOI:10.1049/cp.2013.0298

[5] T. Wen-Long, L. Gao-Peng, and X. Rong-Qing, "Ionospheric clutter mitigation for high-frequency surface-wave radar using two-dimensional array and beam space processing," IET Radar Sonar Navig., vol. 6, no. 3, pp. 202-211, 2012. DOI:10.1049/iet-rsn.2011.0121

[6] Y. Su, Y. Wei, R. Xu, and Y. Liu, "Ionospheric clutter suppression using 
wavelet oblique projecting filter," IEEE Conference on Radar 2017, pp. 1552 1556, 2017. DOI:10.1109/radar.2017.7944454

[7] M. Ravan, R. J. Riddolls, and R. S. Adve, "Ionospheric and auroral clutter models for HF surface wave and over-the-horizon radar systems," Radio Sci., vol. 47, no. 3, pp. 1-12, 2012. DOI:10.1029/2011rs004944

[8] X. L. Chu, J. Zhang, J. I. Yong-Gang, et al., "The second order spectrum extraction from high frequency ground radar sea echoes based on the outline of the RD spectrum," Adv. Marine Sci., 2016.

[9] B. Wang, G. Zhang, L. I. Zhi, et al., "Wavelet threshold denoising algorithm based on new threshold function," J. Comput. Appl., vol. 34, no. 5, pp. 14991502, 2014.

[10] W. H. Zhu, W. An, L. H. You, et al., "Wavelet threshold denoising algorithm based on improved wavelet threshold function," Appl. Comput. Syst., vol. 25, no. 6, pp. 191-195, 2016.

[11] A. M. Atto, D. Pastor, and G. Mercier, "Wavelet shrinkage: Unification of basic thresholding functions and thresholds," Signal Image Video Process., vol. 5, no. 1, pp. 11-28, 2011. DOI:10.1007/s11760-009-0139-y

\section{Introduction}

The use of high frequency surface wave radar (HFSWR) in the technology of remote sensing $[1,2]$ for detecting the ocean can be seen in recent years, the sea state information associated with the waves is mainly included in the two spectral range of the radar echo spectrum, the existing ocean wave information acquisition technology mainly aims at the analysis and processing of the second-order spectrum and the extraction of the characteristic parameters to obtain the related ocean information, such as the effective wave height and the ocean wave spectrum $[3,4]$. HFSWR is affected by ionospheric clutter with different morphological characteristics in time, space and frequency bands, it shows obvious non-stationary signal characteristics in frequency spectrum, and its energy is so strong that it often completely drowns out useful ocean echo spectrum [5, 6, 7]. So it is difficult to extract the second-order spectrum of ocean echo on the distance element with ionospheric signal interference, and then it is a great obstacle to the subsequent sea state information retrieval. Therefore, how to separate the second-order spectra from the ionospheric echo spectrum effectively is the key to obtain the sea state information of the region. To resolve the above problem, this paper proposes a hybrid echo signal processing algorithm. The method of dividing the second-order spectral region under ionospheric clutter is studied in-depth, and the validity of the algorithm is verified by the measured data. The new algorithm solves the problem of two order spectrum extraction under ionospheric interference, and improves the extraction accuracy, which lays the foundation for the next research.

\section{The present situation of second-order spectrum extraction}

In the Doppler spectrum of radar signal echo, the second-order spectrum is usually distributed on both sides of the first order spectrum. The signal-to-noise ratio (SNR) is obviously lower than that of the first order spectrum, and has a certain spectrum range. Because of the noise, clutter and other kinds of interference signals in 
Doppler spectrum, the difficulty of extracting second-order spectra is increased. At present, the extraction of the second-order spectra is mostly aimed at the Doppler spectrum without ionospheric disturbances and it is extracted by constructing range Doppler (RD) spectral contour [8]. However, when dealing with the Doppler spectrum with ionospheric disturbances, the interference signal has obvious influence on the outline of the RD spectrum, and cannot reflect the general location of the second-order spectrum.

\section{Second-order spectrum extraction under ionospheric clutter}

Since the study of second-order spectrum extraction usually takes place without the ionosphere interference, in the case of ionospheric disturbances, the previous extraction methods have great limitations, for example, the peaks of the secondorder spectrum and the second-order spectral boundary cannot be determined, and the second-order spectrum is completely disturbed by the ionosphere. In order to solve the second-order spectrum extraction in the background of ionospheric interference, this paper attempts to improve the probability and accuracy of the second-order spectrum extraction from the spectrum of ionospheric interference from three directions as follows.

\subsection{The prolongation of coherent time}

In order to improve the accuracy of the second-order spectrum extraction, the paper pretreated the echo data and extended the coherence length of the data to achieve the purpose of broadening the spectrum. According to the initial coherence time of the echo data, the cumulative increment of the integer double data acquisition time is performed.

$$
\mathrm{N}=t * k
$$

In the upper formula, $\mathrm{N}$ is the coherence length of the extended data, and $\mathrm{t}$ is the minimum cumulative time, and $\mathrm{k}$ is the coherent multiple.

\subsection{The cancellation of ionospheric clutter}

In HFSWR echo data, the first-order peak signal information mainly contained in the low frequency component of the scale coefficient, the ionospheric echo information is present in the high frequency component of the wavelet coefficients. The wavelet threshold contraction algorithm [9] will deal with the interference signal with strong correlation in the wavelet coefficients, and it will be normalized to the elimination result.

Similar to [10], the threshold function uses the following expression:

$$
y_{p}(x)= \begin{cases}\operatorname{sgn}(x) \sqrt[j]{\left(|x|^{j}-\lambda^{j}\right),} & |x| \geq \lambda \\ 0, & |x| \leq \lambda\end{cases}
$$

Adopted from [11], the threshold selection uses the following expression:

$$
\lambda=\frac{\sigma \sqrt{2 \ln (N)}}{\ln (j+1)}
$$

$\mathrm{j}$ is the decomposition scale. 


\subsection{The choice of threshold for signal-to-noise ratio of second-order spectrum boundaries}

The threshold is used to judge the boundary of the second-order spectrum, and the selection of the threshold must have some ambiguity after the ionospheric elimination operation. In order to solve the problem of threshold ambiguity, applying two order boundary spectrum average value dynamic calibration of adjacent distance element without ionospheric disturbance, and reference spectra of the first substrate noise and signal-to-noise ratio, to determine the second-order spectrum threshold.

$$
S N R_{\text {ave }}=\frac{1}{n} \sum_{i=1}^{n} S N R_{i}
$$

$\mathrm{SNR}_{\mathrm{ave}}$ is the mean value of the second-order spectrum boundary; $\mathrm{SNR}_{\mathrm{i}}$ is the signal-to-noise ratio of the ith data for the second-order spectrum boundaries.

\section{Second-order spectrum extraction and analysis}

The experimental data of this paper are taken from the spectrum of a distance element at 11 am of September 26, 2013 in the Beibu Gulf $\left(08^{\circ} 13.30^{\prime} \mathrm{E}, 21^{\circ}\right.$ $30.30^{\prime} \mathrm{N}$ ) as shown in Fig. 1(a).

\subsection{Coherent accumulation of ionospheric elimination}

In the time to eliminate the interference on the ionosphere, considering the ionospheric cancellation algorithm that will cause loss of signal-to-noise ratio for the echo spectrum data, due to less number of points in every game, it is easy to cause the cancellation after the second-order spectrum is not obvious or cancellation of the elimination of the ionosphere, which is on the order of two subsequent extractions caused by the spectrum great influence. In order to deal with this problem, this paper uses the method of increasing coherent accumulation time, and uses more coherent data to reduce the influence of the ionospheric cancellation algorithm on the second-order spectrum.

From the comparison of Fig. 1(b) (c), it is observed that the spectrum of the data at 256 sampling points is significantly less than that of the 1024 sampling points after coherent integration. In the process of ionospheric elimination, it is obvious that the possibility of extracting two order spectra before coherent accumulation is relatively small. However, after the coherent accumulation, although the ionospheric cancellation algorithm has affected the signal-to-noise ratio of the two order spectrum, the range of the approximate data points contained in the two order spectrum can be distinguished from the spectrum, which reflects the advantages of the more data sampling points. 


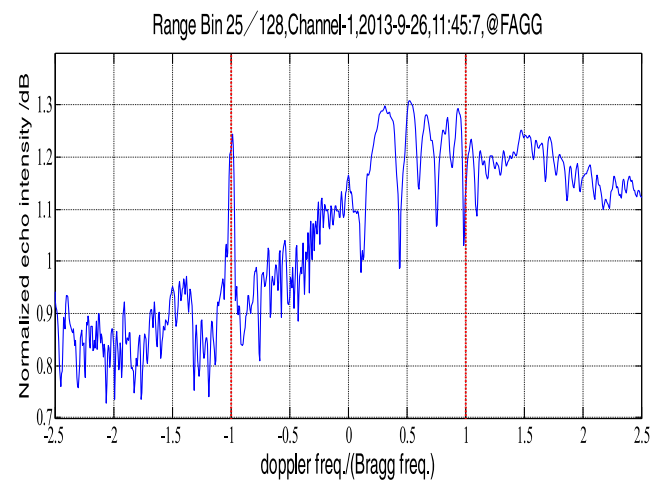

(a)

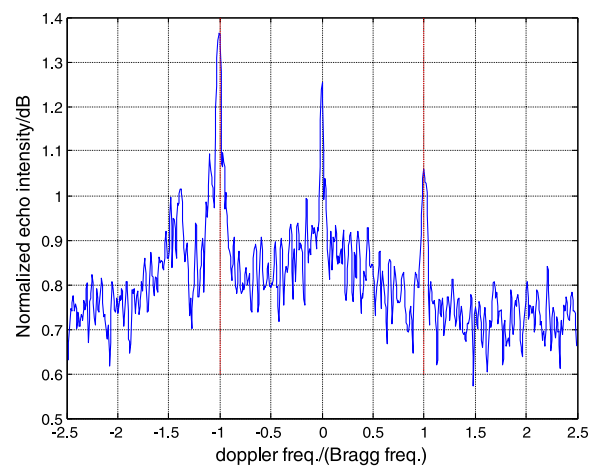

(b)

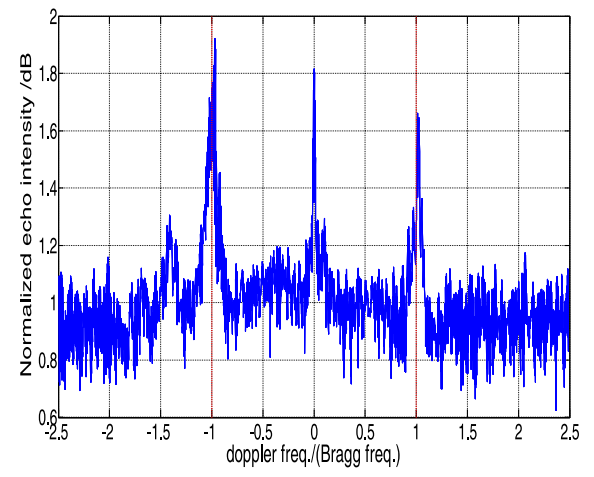

(c)

Fig. 1. The spectra of two groups before and after coherence

\subsection{The extraction of second-order spectrum extraction parameters}

After the coherent accumulation, the signal-to-noise ratio of the spectrum changes with the ionosphere elimination. In this paper, according to the state parameters of the ocean echo spectrum of the distance elements which are not affected by the ionospheric disturbances as the reference, the two order spectra are divided after the ionospheric disturbances are eliminated. The Table I gives the reference mean values of the related main parameters of adjacent interference free range elements, and takes the transformation data before and after the first peak cancellation as the reference basis. The acquisition of the table data parameters is calculated by the echo data received within one hour, and two distance elements and two adjacent distance elements are calculated.

\subsection{Extraction results}

According to the statistics of the above parameters, echo spectrum correlation parameters are based on adjacent undisturbed range elements, and combined with the influence of ionospheric cancellation algorithm on SNR of echo spectrum, ultimately they determine the ionosphere on consumption, increase the coherent time spectrum of second-order boundary spectral signal-to-noise ratio, and then the scope of second-order spectrum is determined, finally, according to the peak parameters of second-order spectrum, the first-order peak area and two spectral region is separated. The Fig. 2 is the result of the second-order spectral partition of the coherent accumulation time spectrum after the above processing. 
Table I. Reference values of related parameters

\begin{tabular}{|c|c|c|c|c|c|c|}
\hline $\begin{array}{c}\text { DI-LSNR } \\
(\mathrm{dB})\end{array}$ & $\begin{array}{c}\text { DI-RSNR } \\
(\mathrm{dB})\end{array}$ & $\begin{array}{c}\text { D-N } \\
(\mathrm{dB})\end{array}$ & $\begin{array}{c}\text { LSNRave } \\
(\mathrm{dB})\end{array}$ & $\begin{array}{c}\text { RSNRave } \\
(\mathrm{dB})\end{array}$ & $\begin{array}{c}\text { LBSNRave } \\
(\mathrm{dB})\end{array}$ & $\begin{array}{c}\text { RBSNRave } \\
(\mathrm{dB})\end{array}$ \\
\hline 6.96 & 14.22 & 1.98 & 33.66 & 40.09 & 17.28 & 12.7 \\
\hline 7.42 & 11.74 & 4.75 & 32.57 & 37.24 & 10.78 & 13.73 \\
\hline 6.67 & 10.76 & 5.34 & 36.78 & 17.63 & 28.79 & 3.83 \\
\hline 8.05 & 13.97 & 3.07 & 31.13 & 14.73 & 14.48 & 2.74 \\
\hline 7.14 & 14.07 & 3.84 & 29.60 & 12.40 & 12.75 & 8.64 \\
\hline 7.59 & 11.32 & 4.91 & 36.07 & 17.82 & 23.63 & 6.44 \\
\hline 5.73 & 15.48 & 1.79 & 35.97 & 18.52 & 21.48 & 11.43 \\
\hline 9.03 & 15.02 & 2.65 & 35.64 & 17.86 & 28.27 & 12.13 \\
\hline
\end{tabular}

DI-LSNR: Difference of SNR of left first order peak after clutter suppression. DI-RSNR: Difference of SNR of right first order peak after clutter suppression. D-N: Noise base difference before and after interference suppression. LSNRave: Mean value of SNR of left first order peak without interference. RSNRave: Mean value of SNR of right first order peak without interference. LBSNRave: Mean value of SNR of left boundary without interference. RBSNRave: Mean value of SNR of right boundary without interference.

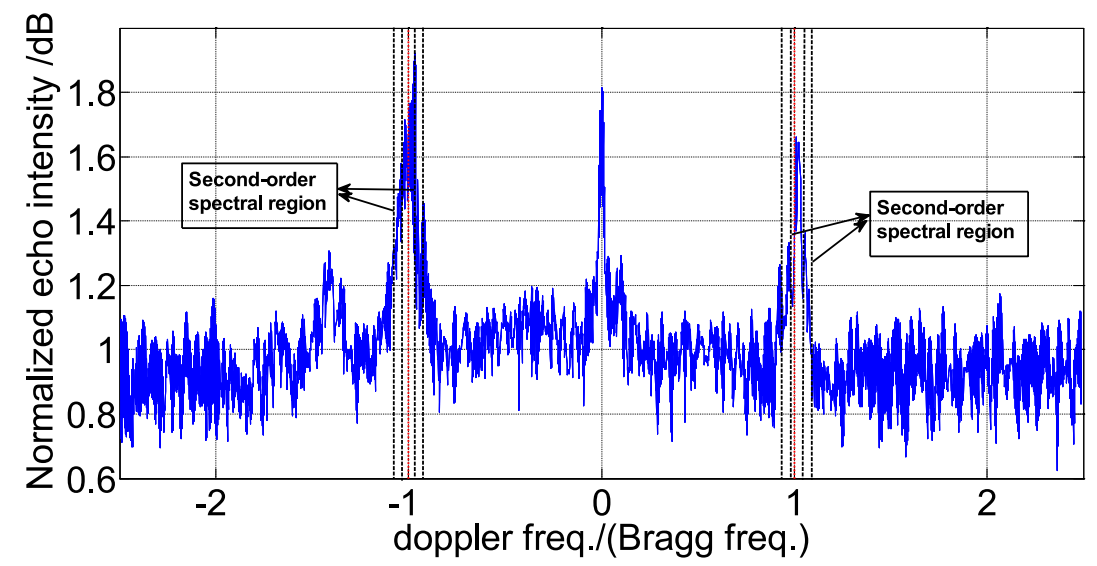

Fig. 2. The result of two order spectrum extraction

\section{Conclusion}

Ionospheric disturbances are one of the most difficult problems to be solved in the radar signal analysis, just in a resource limited environment, analysis of echo signal of the ionospheric disturbance under the conditions of the ionosphere and the elimination of the echo spectrum in a certain extent. In this paper, the good weather conditions and the characteristics of the states in a specific area are basically the same, by increasing the coherent time, and combining with the spectrum parameters of adjacent distance elements without ionospheric disturbances, we develop the second-order spectrum feature analysis and extraction using echo spectrum with ionospheric interference by mathematical statistical analysis and approximate method. Finally, the calculation results show that the echo spectrum of ionospheric disturbances can be analyzed effectively and reasonably based on certain ocean condition and the stability principle. 


\section{Acknowledgment}

The authors would like to thank many teachers for useful suggestions and discussions. This work was partially supported by the National Natural Science Foundation of China (61661009) and Innovation Project of Guangxi Graduate Education (YCSW2017056). 